\title{
Achieving Proportional Fairness via AP Power Control in Multi-Rate WLANs
}

\author{
Wei Li, Yong Cui, Member, IEEE, Xiuzhen Cheng, Member, IEEE, Mznah A. Al-Rodhaan, \\ and Abdullah Al-Dhelaan
}

\begin{abstract}
In this paper, we consider how to achieve proportional fairness in multi-rate 802.11 WLANs by investigating an integrated problem of power control and AP Association in order to provide an effective tradeoff between network throughput and fairness. Since jointly considering power control and AP association for proportional fairness is NP-hard, we propose a centralized heuristic approach. By introducing a new concept of AP utility, we establish the relationship between the network utility and the AP utility according to proportional fairness. This relationship is exploited to design an algorithm PCAP to optimize the network utility by increasing the average and decreasing the variance of the $A P$ utility. Extensive simulation study is performed and the results demonstrate that PCAP yields a significant improvement in terms of throughput, fairness, and power consumption compared to other popular power control algorithms.
\end{abstract}

Index Terms-AP association, power control, proportional fairness, multi-rate WLANs.

\section{INTRODUCTION}

I $\mathrm{N}$ recent years, the density of IEEE 802.11 WLAN deployments has been dramatically increased. A higher density offers a shorter distance from APs to users, which allows the use of higher transmission rates. However, densely-deployed WLANs face some challenges. On one hand, each user associates with the AP with the strongest received signal strength indicator (RSSI) by default. Since users are not uniformly distributed among APs, some APs tend to suffer heavy loads while adjacent APs may carry very light traffic or even be idle. Load imbalance may cause bandwidth starvation of some users, leading to a sharp decline in the network throughput. On the other hand, surveys of typical deployments in the US indicate that the default transmit power levels of the APs and the users are often set to the maximum without considering the coverage of APs and the distance of users to APs [1]. Such a default policy results in a higher interference

Manuscript received October 26, 2010; revised May 17, 2011; accepted July 31,2011 . The associate editor coordinating the review of this paper and approving it for publication was T. Hou.

W. Li is with the Department of Computer Science, The George Washington University, USA (e-mail: weili@gwmail.gwu.edu).

Y. Cui is with the Department of Computer Science, Tsinghua University, Beijing, P. R. China (e-mail: cuiyong@tsinghua.edu.cn).

$\mathrm{X}$. Cheng (corresponding author) is with the Department of Computer Science, The George Washington University, Washington DC, USA, and the College of Computer and Information Sciences, King Saud University, Riyadh, Saudi Arabia (e-mail: cheng@gwu.edu).

M. A. Al-Rodhaan and A. Al-Dhelaan are with the College of Computer and Information Sciences, King Saud University, Riyadh, Saudi Arabia (email: \{rodhaan, dhelaan\}@ksu.edu.sa).

This work has been supported by the National Science Foundation of the US (CNS-0831852) and the NPST program by King Saud University (Project No. 10-INF1184-02).

Digital Object Identifier 10.1109/TWC.2011.091411.101899 among overlapping APs. Motivated by these observations, we investigate AP power control in this paper to provide an effective tradeoff between aggregated network throughput and fairness.

The popular 802.11 MAC protocol provides equal long-term transmission opportunities to all users such that the users with the same frame size achieve equal throughput (i.e. throughputbased fairness) [2]-[5]. When the users transmit at the same bit rate, 802.11 also achieves time-based fairness [5], i.e., each user obtains an equal share of the channel occupancy time. Nevertheless, this is not true for multi-rate WLANs, in which users with lower bit rates occupy the channel for longer time than those with higher bit rates, drastically reducing the network throughput [2], [3]. Recent studies indicate that timebased fairness outperforms throughput-based fairness in multirate WLANs [2], [6].

We also have two fairness criteria that are widely employed in network resource management: max-min fairness [7], which distributes throughput as equally as possible via maximizing the minimum throughput among all users, and proportional fairness [4], which allocates bandwidth to users in proportion to their bit rates to maximize the sum of the bandwidth utilities of the users. Our prior study [8] indicates that proportional fairness and time-based fairness are equivalent in multi-rate WLANs when all users have the same weight. The equivalence of max-min fairness and throughput-based fairness under the same condition (integral association) is proved in [9]. It is also argued [4], [10] that proportional fairness provides a good tradeoff between network throughput and fairness.

According to IEEE 802.11, AP transmit powers can be adjusted in an allowable range. This technique is called power control, which helps to conserve battery energy in mobiles and to mitigate interference. Different power control schemes [1], [11], [12] have been investigated, but they all assume that the user-AP association remains unchanged when power is adjusted. Nevertheless, an 802.11 user by default selects the AP with the strongest RSSI. Thus power control may cause user-AP association change. This phenomenon has been investigated by cell-breathing techniques [13], [14], which separate the AP transmit power into beacon power and data power, and only the beacon power is adjusted for user association control to balance the AP loads. Since beacon power adjustment results in the expansion and shrinkage of the coverage of an AP (a "cell"), such a technique is called "cell-breathing". As a contrary, our study focuses on the data power control to achieve proportional fairness.

In this paper, we propose a power control algorithm termed PCAP, which stands for Power Control for AP Performance 
enhancement, to simultaneously consider power control and AP association for proportional fairness in multi-rate WLANs. We first introduce the concept of the AP utility and establish the relationship between the AP utility and the network utility according to proportional fairness. Based on the arithmeticgeometric mean inequality, we then prove that maximizing the network utility can be achieved by maximizing the average and minimizing the variance of the AP utility. According to this result, we design the algorithm PCAP, which consists of two sub-algorithms MAP and MPV. MAP, which stands for Maximize Average Performance, intends to maximize the average AP utility. MPV, which stands for Minimize Performance Variance, intends to minimize the variance of the AP utility. MAP and MPV adjust the AP utility by power control, which might trigger user-AP association changes. In our consideration, AP association adopts the default rule, i.e., the strongest RSSI. Our simulation study confirms that the proposed algorithms can improve the network utility, reduce the power consumption, and achieve proportional fairness.

There are three major contributions in this paper:

- We establish the relationship between the network utility and the AP utility according to proportional fairness, which helps us to optimize the network utility.

- We propose and validate the algorithm PCAP, which includes MAP and MPV, to achieve proportional fairness via AP power control in multi-rate WLANs. PCAP does not require special mechanisms for association control.

- Our simulation study indicates that PCAP outperforms popular power control algorithms such as MARL [15], SR [12], and SSF [10], in terms of network utility and aggregated throughput, Jain's fairness index, and power consumption.

The rest of the paper is organized as follows. Related work is discussed in Section II. Network model is introduced in Section III. The impact of power control on the network performance is analyzed in Section IV. The algorithms are described in detail in Section V. After presenting our simulation results in Section VI, we conclude this paper in Section VII.

\section{RELATED WORK}

A wide range of transmit power control algorithms have been proposed in recent literature to improve the network performance and reduce the interference in 802.11 wireless networks.

In [1], a power control algorithm called PERF is proposed to decrease the transmit power of an AP as long as it can support the highest transmission rates of its users. Such a greedy method is unlikely to be optimal from the viewpoint of the overall network performance. Mhatre et al. [11] establish sufficient conditions for starvation-free power control, and propose a power control algorithm that assigns higher transmit powers to the more heavily loaded APs. This algorithm adopts a cross-layer approach that can jointly tune the transmit power and the carrier sense parameters of the MAC layer to maximize the sum of the long term throughput, without any consideration of fairness. Hasu and Koivo [12] utilize transmit power control to allocate transmission rates in cellular radio systems. They first compute the feasible rates of users by estimating a spectral radius of any non-negative channel gain matrix and then calculate the transmit powers based on the relationship between power and rate.

Qian et al. [15] propose a novel algorithm for the nonconvex power control optimization problem. In their approach, the problem is transformed into a monotonic optimization problem whose optimal solution always bounds the feasible $(1+$ SINR) region. A joint power control and scheduling problem is considered in clustered multi-hop TD/CDMA wireless ad hoc networks [16], in which a cluster based architecture is introduced to provide a centralized control within a cluster, and the corresponding power control and scheduling scheme is derived to maximize a network utility function and guarantee the minimum rate required by each traffic session. Note that this consideration focuses on intra-cluster traffic transmissions where a central controller is available. Chiang et al. [17] formulate the power control problem with geometric programming and present a distributed geometricprogramming-based solution. A drawback of this approach is that geometric programming is applicable only when the Signal-to-Interference Ratio (SIR) is much larger than $0 \mathrm{~dB}$. Therefore it is not suitable for the low SIR case.

Game theory has been exploited to tackle the power control problem in wireless networks. To achieve the maximum throughput with a minimum energy consumption, Chen et al. [18] carry out their studies using game theory for three specific games: the fixed-rate power control game, the fixed-power rate control game, and the joint power rate control game. For the case of cooperative APs, Song et al. [19] present a negotiation-based throughput maximization algorithm that automatically adjusts the operating channels and power levels among access points from a game theoretical perspective. Rasti et al. [20] propose a Pareto-efficient and distributed power control scheme for wireless networks, which employs a noncooperative game-theoretic approach to derive a novel pricing scheme that is linearly proportional to SIR. According to the distributed price setting [20], users update their transmit powers for different goals such as fairness and aggregated throughput optimization.

This paper proposes PCAP to jointly consider power control and AP association for proportional fairness in multirate WLANs. We first consider the relationship between the AP utility and the network utility. This relationship is then exploited in PCAP to adjust the transmit powers of the APs to provide a better network utility. Our objectives are achieved by two greedy algorithms MAP and MPV, which intend to maximize the average AP utility and minimize the variance of the AP utility, respectively. As network utility is a metric that provides a tradeoff between throughput and fairness, PCAP provides a better bandwidth allocation to all users in terms of proportional fairness while still maintains a high throughput. Compared to the power control mechanisms surveyed above, PCAP has the following advantages:

- Enhanced network throughput. Power control and AP association mutually influence each other, i.e., power adjustment could make a user re-associate to a better AP. Jointly considering both power control and APAssociation can help to obtain a higher network throughput. 
- Fair bandwidth allocation. One design objective of PCAP is to maximize the total utility of the user bandwidths, which can avoid starving the users with lower bit rates.

\section{Network Model and Problem Formulation}

Our network topology models an IEEE 802.11 based high density WLAN, which contains multiple APs. Each AP has a limited coverage area, with all the areas forming the network coverage area. We assume that overlapping coverage areas of adjacent APs may exist. We further assume that each user resides in at least one AP's coverage area so as to obtain the Internet service. A user is allowed to choose only one AP if it is in an overlapping coverage area whereas each AP can serve multiple users at the same time.

In this paper, we consider the case when all APs work on the same channel. Since in many real-world applications such as social networking [1], [10], [11], [13], [19], downlink produces the dominant traffic in the whole network, we focus on the downlink only. Under such an assumption, a user in the overlapping coverage area is mainly interfered by other APs. The bit rate of a user is determined by the received powers of all the links from the APs covering the user. Let $\gamma_{i j}$ denote the SINR of user $i$ when associated with AP $j$. We have,

$$
\gamma_{i j}=\frac{g_{i j} p_{j}}{\sum_{k \in A_{i} \cap k \neq j} g_{i k} p_{k}+N_{0}},
$$

where $g_{i j}$ is the channel gain from AP $j$ to user $i, p_{j}$ is the transmit power of AP $j, N_{0}$ is the additive Gaussian white noise, and $A_{i}$ is the set of APs covering user $i$. The range of the transmit power $p_{j}$ is denoted by $\left[p_{\min }, p_{\max }\right]$, where $p_{\min }$ and $p_{\max }$ are the minimum and the maximum allowed powers, respectively. We assume that each AP is configured to use one of $L$ transmit power levels, with $p_{\min }$ corresponding to level 1 and $p_{\max }$ corresponding to level $L$. The power of level $l, l=1,2, \cdots, L$, is $\alpha$ times stronger than that of level $l-1$, where $\alpha$ is defined as:

$$
\alpha=\sqrt[L-1]{p_{\max } / p_{\min }}
$$

Note that similar discrete transmit power models have been adopted by previous research [13]. The IEEE 802.11 standard specifies the range of the transmit powers but does not give a clear definition on the power levels. We adopt (2) for its simplicity.

TABLE I

THE RELATIONSHIP BETWEEN SINRS AND RATES

\begin{tabular}{|c|c|c|c|c|c|c|c|c|}
\hline$\gamma_{i j}(\mathrm{~dB})$ & $6-7.8$ & $7.8-9$ & $9-10.8$ & $10.8-17$ & $17-18.8$ & $18.8-24$ & $24-24.6$ & $24.6-$ \\
\hline$v_{i j}(\mathrm{Mbps})$ & 6 & 9 & 12 & 18 & 24 & 36 & 48 & 54 \\
\hline
\end{tabular}

According to 802.11 , there are eight bit rate levels in the network. Each bit rate level is determined by a range of SINR, which is shown in Table I [21], and expressed by (3):

$$
v_{i j}=f(G, P),
$$

where $v_{i j}$ is the bit rate between user $i$ and $\operatorname{AP} j, G$ is the channel gain matrix with the rows representing users and the columns representing APs, and $P$ is the vector of transmit powers.
In this paper, we adopt the default IEEE 802.11 AP association method, i.e., a user always selects the AP with the strongest received signal. The association coefficient $x_{i j}$ is a $0-1$ binary variable that is equal to 1 if and only if user $i$ associates to $\mathrm{AP} j$. Let $b_{i}$ be the effective bandwidth allocated to user $i$, i.e., $b_{i}=\sum_{j=1}^{n} x_{i j} t_{i j} v_{i j}$, where $t_{i j}$ is the effective transmission time between user $i$ and AP $j$ within one unit of time (to be defined). Assume that users are greedy and always download data during their transmission time. Given a user-AP association, our goal is to allocate each user a sufficient amount of effective bandwidth in a proportional manner without unduly restricting the amount of effective bandwidths available to others. According to proportional fairness [4], the network utility, $U$, is defined to be the total utility of the user bandwidths, which is the sum of the logarithms of the bandwidths allocated to all users:

$$
U=\sum_{i=1}^{m} w_{i} \log \left(b_{i}\right)=\sum_{i=1}^{m} w_{i} \log \left(\sum_{j=1}^{n} x_{i j} t_{i j} v_{i j}\right),
$$

here $m$ and $n$ are the number of users and APs, respectively, $w_{i}$ is the weight of user $i$. Prior study [10] indicates that the value $U$ provides a good trade-off between the network throughput and fairness.

A unit of time in which the network is stable, with no new user joins and no current user leaves, is to be considered. A user is allowed to choose one and only one AP within the unit time. Each AP assigns a fraction of its transmission time to a user in accordance with proportional fairness. As articulated by our prior research [8], the transmission time between user $i$ and AP $j$ can be obtained from (5) given an integral user-AP association and the user weights.

Theorem 1: [8] Given an integral user-AP association matrix $X=\left\{x_{i j}\right\}$, the unique optimal transmission time assigned to user $i$ by AP $j$ according to proportional fairness is

$$
t_{i j}=\frac{w_{i} x_{i j}}{\sum_{k=1}^{m} w_{k} x_{k j}} .
$$

Mathematically, we can formulate the following non-linear program for our integrated problem of AP Association and Power Control.

$$
\begin{aligned}
\max & \sum_{i=1}^{m} w_{i} \log \left(b_{i}\right)=\sum_{i=1}^{m} w_{i} \log \left(\sum_{j=1}^{n} x_{i j} t_{i j} \cdot f(G, P)\right) \\
\text { s.t. } & \sum_{j=1}^{n} x_{i j}=1,1 \leq i \leq m, \\
& \sum_{i=1}^{m} x_{i j} t_{i j} \leq 1,1 \leq j \leq n, \\
& x_{i j} \in\{0,1\}, 1 \leq i \leq m, 1 \leq j \leq n \\
& p_{j} \in\left\{p_{\min }, \cdots, p_{\max }\right\}, 1 \leq j \leq n .
\end{aligned}
$$

(6) is referred as the Integrated Optimization for Proportional Fairness (IOPF). The constraint (6.b) indicates that each user can associate with one and only one AP at any time; the constraint (6.c) requires that the total transmission time of each AP $j$ can not exceed 1; the constraint (6.d) assures that $x_{i j}$ is a binary variable that is equal to 1 if and only if the user $i$ 
is associated with the AP $j$; the constraint (6.f) specifies the range of the variable $p_{j}$. We can show that IOPF is NP-hard by slightly adapting the reduction procedure in [22]. On the other hand, a special case of IOPF, AP-association based on proportional fairness when AP powers are fixed, is proved to be NP-hard in [10], which implies the NP-hardness of IOPF.

\section{Power Control and Performance}

\section{A. The Impact of Power Control on Network Performance}

Power control is an effective approach to improve the network throughput [1], [15]. Nevertheless, adjusting transmit powers may cause AP re-association because a user selects another AP immediately once its original AP signal is not the strongest. In this subsection, we analyze the impact of power control on the network utility.

Definition 1: A network state $S$ is defined as the dual group of the association matrix $X$ and the AP transmit power vector $P$, i.e. $S=(X, P)$.

Theorem 2: If all APs increase (or decrease) equal number of power levels simultaneously, the user-AP association in the network does not change.

Proof: Without loss of generality, we assume that all APs increase their transmit powers simultaneously by $l$ levels and use $\gamma_{i j}^{\prime}$ to denote the SINR after the change. Let $p_{j}$ be the transmit power before the adjustment. From (1) and (2), we have

$$
\begin{aligned}
\gamma_{i j}^{\prime} & =\frac{g_{i j} p_{j} \cdot \alpha^{l}}{\sum_{k \in \mathbf{A}_{\mathbf{i} \cap k \neq j}} g_{i k} p_{k} \cdot \alpha^{l}+N_{0}} \\
& =\frac{g_{i j} p_{j}}{\sum_{k \in \mathbf{A}_{\mathbf{i} \cap k \neq j}} g_{i k} p_{k}+N_{0} / \alpha^{l}} .
\end{aligned}
$$

(7) indicates that for all APs, increasing (or decreasing) equal power levels is equivalent to decreasing (or increasing) the background noise experienced by the user. Obviously, the AP with the strongest received signal remains unchanged for a user before and after the adjustment. Thus, the use-AP association in the network does not change.

From Table I, we observe that the bit rate is 0 if the SINR is less than $6 \mathrm{~dB}$, and is $54 \mathrm{Mbps}$ if the SINR is more than 24.6 $\mathrm{dB}$. Between the minimum SINR and the maximum SINR, (3) and (7) indicate that the bit rate is non-decreasing with the increase in the SINR when $l \geq 0$. That is,

$$
\begin{aligned}
v_{i j}^{\prime} & =f\left(G, \alpha^{l} P\right) \\
& =f\left(\frac{g_{i j} p_{j} \cdot \alpha^{l}}{\sum_{k \in \mathbf{A}_{\mathbf{i}} \cap k \neq j} g_{i k} p_{k} \cdot \alpha^{l}+N_{0}}\right) \\
& =f\left(\frac{g_{i j} p_{j}}{\sum_{k \in \mathbf{A}_{\mathbf{i}} \cap k \neq j} g_{i k} p_{k}+N_{0} / \alpha^{l}}\right) \\
& \quad f\left(\frac{g_{i j} p_{j}}{\sum_{k \in \mathbf{A}_{\mathbf{i}} \cap k \neq j} g_{i k} p_{k}+N_{0}}\right) \\
& =f(G, P)=v_{i j} .
\end{aligned}
$$

From (8), we can see that the bit rate of the users is nondecreasing when increasing all the transmit powers simultaneously for a fixed user-AP association. Thus, the network utility is also non-decreasing. Furthermore, we observe that with such an adjustment, the improvement of the network utility is not significant.

\section{B. Network Utility vs. AP Utility}

This paper intends to improve the network utility from APs' point of view. Our basic idea comes from the arithmeticgeometric mean inequality. For convenience, we first introduce the concept of AP utility, which is defined to be the weighted bandwidth product of all users associated with the AP.

Definition 2: The utility of $A P j$, denoted by $U_{j}^{a}$, is defined by $U_{j}^{a}=\prod_{i \in C_{j}} b_{i}^{w_{i}}$, where $C_{j}$ denotes the set of users associated with AP $j$.

Definition 3: The average AP utility in a network is defined as the mean of the utilities of all APs: $\overline{U^{a}}=\frac{1}{n} \sum_{j=1}^{n} U_{j}^{a}$.

Definition 4: The utility deviation of $A P j$ is defined as: $\Delta U_{j}^{a}=U_{j}^{a}-\overline{U^{a}}$.

Definition 5: The maximum utility deviation in the network is defined as: $\Delta U_{\max }^{a}=\max \left\{\left|\Delta U_{j}^{a}\right|, 1 \leq j \leq n\right\}$.

Definition 6: The variance of the AP utility in the network is defined as: $\sigma_{u}^{2}=\frac{1}{n} \sum_{j=1}^{n}\left(U_{j}^{a}-\overline{U^{a}}\right)^{2}$.

From Definition 2, it can be seen that the AP utility is related to not only the number of users, but also the user effective bandwidths. This provides an efficient tradeoff between the load of the AP and the user bandwidth. Obviously, for a given user-AP association, the total utility of the user bandwidth is equivalent to that of the AP. The relationship between the network utility $U$ and the AP utility can be expressed by the following equation.

$$
\begin{aligned}
U & =\sum_{i=1}^{m} w_{i} \log \left(b_{i}\right)=\sum_{j=1}^{n}\left(\sum_{i \in C_{j}} w_{i} \log \left(b_{i}\right)\right) \\
& =\sum_{j=1}^{n} \log \left(\prod_{i \in C_{j}} b_{i}^{w_{i}}\right)=\sum_{j=1}^{n} \log \left(U_{j}^{a}\right) .
\end{aligned}
$$

It is well-known that the geometric mean of $n$ positive real numbers $y_{i}(i=1,2, \cdots, n)$, is not larger than the corresponding arithmetic mean. This arithmetic-geometric mean inequality is expressed by

$$
\sqrt[n]{y_{1} y_{2} \cdots y_{n}} \leq \frac{y_{1}+y_{2}+\cdots+y_{n}}{n},
$$

and the equality holds if and only if $y_{1}=y_{2}=\cdots=y_{n}$.

Since the utility of each AP is positive, we can apply the above inequality to obtain

$$
\begin{aligned}
U & =\sum_{j=1}^{n} \log \left(U_{j}^{a}\right)=\log \left(\prod_{j=1}^{n} U_{j}^{a}\right) \\
& \leq n \log \left(\frac{1}{n} \sum_{j=1}^{n} U_{j}^{a}\right)=n \log \left(\overline{U^{a}}\right) .
\end{aligned}
$$

Eq. (11) describes the relationship between the network utility $U$ and the average AP utility $\overline{U^{a}}$, and $U=n \log \left(\overline{U^{a}}\right)$ if and only if $U_{1}^{a}=U_{2}^{a}=\cdots=U_{n}^{a}=\overline{U^{a}}$ for a given $\overline{U^{a}}$. Note that $n \log \left(\overline{U^{a}}\right)$ serves as an upper-bound of $U$, and this upper-bound is achieved when all AP utilities equals the average AP utility. Thus, maximizing the network utility can be approximated by maximizing the average AP utility, which 
increases the upper-bound, and minimizing the variance of the AP utility without decreasing the average AP utility, which helps to achieve the upper-bound. In other words, we need to improve the average AP utility while reducing its variance in order to get a better network utility.

\section{Power Control Algorithm}

\begin{tabular}{l} 
Algorithm 1 Power Control for AP Performance Enhancement \\
(PCAP) \\
\hline 1: Initialize the network state; \\
2: Maximize the average AP utility via power control; \\
3: Minimize the AP utility variance via power control. \\
\hline
\end{tabular}

Eq. (11) and the corresponding analysis in Section IV-B indicates that the network utility is maximized when all APs obtain the same utility for a fixed average AP utility. But in reality, forcing all APs to achieve the same utility without decreasing the total AP utility is not possible as the APs usually cover different number of users with different effective bandwidths. These observations motivate us to design our centralized power control algorithm PCAP, shown in Alg. 1, to first maximize the average AP utility and then minimize its variance. Note that maximizing the average AP utility increases the achievable upper-bound of the network utility while minimizing the AP utility variance without decreasing the average AP utility helps the network utility to approach to its upper-bound. PCAP is executed at a network coordinator that will collect the necessary information based on which to calculate the power adjustment. Assuming the availability of a centralized network coordinator is practical as many realworld deployments employ Cisco or Aruba wireless LAN controllers to perform the centralized management [23], [24]. Initially, all APs are assigned with the maximum transmit power and each user selects the AP with the highest RSSI to associate (Step 1). Then we adjust the transmit powers to improve the average AP utility (Step 2). Finally, we perform power control to decrease the variance of the AP utility (Step $3)$. The last two steps are detailed in the next two sub-sections.

\section{A. Maximize the Average Performance}

At the initial network state, all APs use the default maximum transmit power. We need to reduce the transmit powers in order to decrease the interference among APs and improve the average AP utility. Our algorithm, Maximize Average Performance (MAP), iteratively locates a state that yields the maximum average AP utility. At any iteration, we use a function Maximize_Average (MA) to calculate the network state that maximizes the average AP utility. The function MA needs to satisfy the following two conditions:

- Condition 1: The final state of each iteration must be the one that yields the maximum network utility.

- Condition 2: Each iteration should not decrease the average AP utility.

To meet both conditions, we introduce the variables $S^{\prime}, U^{\prime}$, and $\overline{U^{\prime a}}$ to record the network state, the network utility, and the average AP utility, respectively. Let $F$ be the set of APs whose

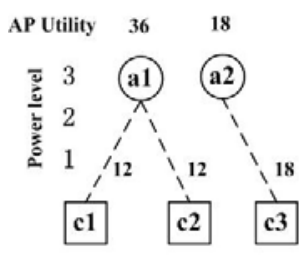

(a) The network initial state

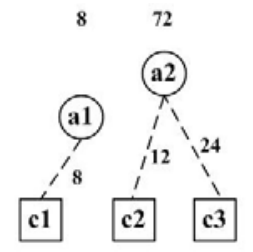

(b) First iteration, $\mathrm{F}=\{\mathrm{a} 2\}$

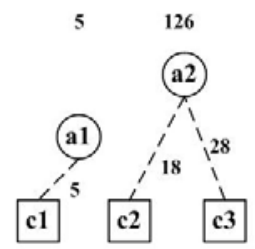

(c) Second iteration, $\mathrm{F}=\{\mathrm{a} 2, \mathrm{a} 1\}$

Fig. 1. An example to illustrate the process of MAP.

transmit powers have already been determined by the previous executions of the algorithm MA. Initially, $F=\emptyset$. Each run of MA finds an AP to be placed in $F$ whose transmit power can not be further adjusted for performance improvement.

The operation of the algorithm MA is executed as follows: We first initialize the recording variables. Then MA locates the AP $k$ with the best AP utility. If $p_{k}>p_{\text {min }}, p_{k}$ is decreased by one level. With this new transmit power of $\mathrm{AP}$, the variables $k, S, U$, and $\overline{U^{a}}$ are updated. If neither the network nor the average AP utility is decreased, AP $k$ takes the new transmit power, and the newly derived values for $S, U$, and $\overline{U^{a}}$ are recorded. The iterative loop of MA terminates only when decreasing the transmit power of the AP with the best AP utility does not increase the average AP utility. This algorithm is described in Alg. 2.

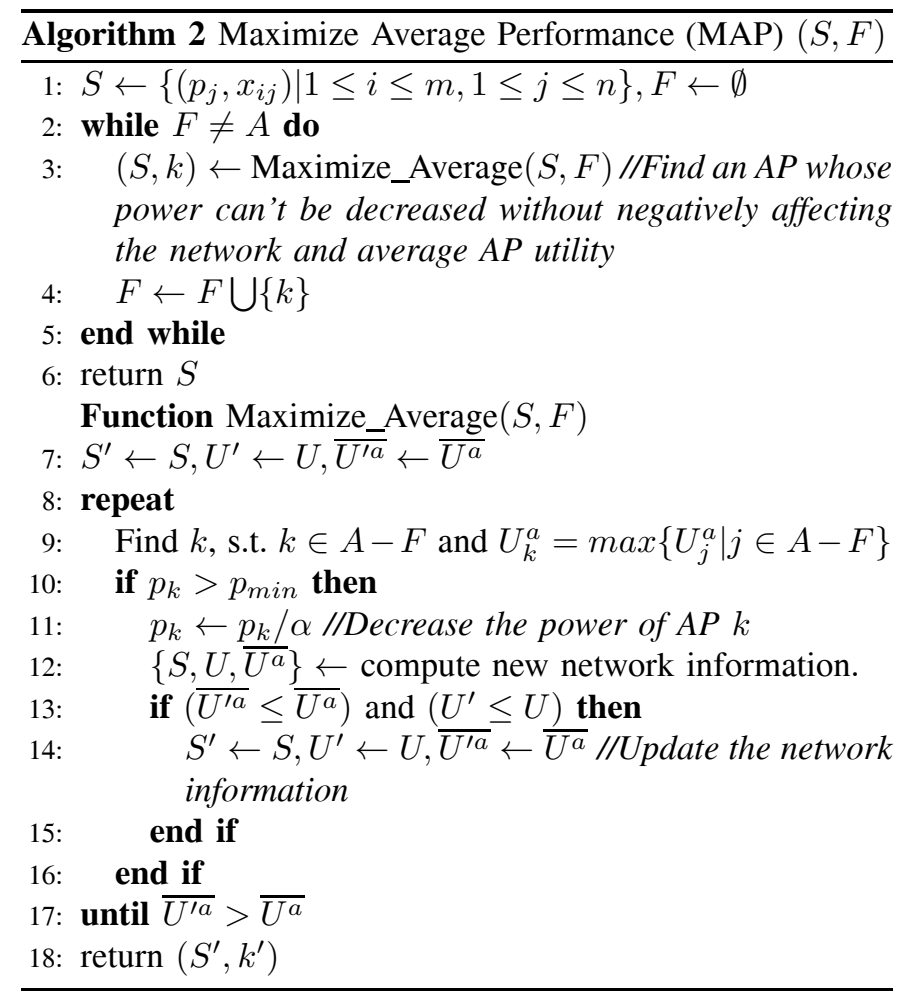

Let $m, n$, and $L$ be the number of users, APs, and power levels, respectively. The while loop in MAP (lines 2-5) calls the MA procedure $n$ times. In MA, lines 9-16 are repeated at most $n L$ times. During each iteration of MA, the computation of the new network information (line 12) takes $m$ time. Therefore the time complexity of MAP is $O\left(m n^{2} L\right)$.

We take the example shown in Fig. 1 to illustrate the process of the MAP algorithm. In Fig. 1, $a_{1}$ and $a_{2}$ are two APs, and 
$c_{1}, c_{2}$ and $c_{3}$ are users with the same weight. There are three power levels in the network. The dashed line between a user and an AP denotes the user-AP association, and the number beside it is the bit rate. Initially, the network utility is 2.6 , the average AP utility is 19.25, and the aggregated throughput is 25.5. The bandwidths of the three users are 5, 4.5 and 16, respectively. In the first iteration, $a_{1}$ has the best performance, and therefore its power is reduced by one level when MA is executed. After reducing the transmit power of $a_{1}, U$ and $\overline{U^{\prime a}}$ become 2.76 and 40, respectively. Since both $U$ and $\overline{U^{\prime a}}$ are improved, we continue looping in MA. Now the AP with the best performance is $a_{2}$. But reducing the transmit power of $a_{2}$ by one level negatively affects the average AP utility. Therefore the algorithm MA terminates, and $a_{2}$ is put in $F$ by MAP. In the next iteration, $a_{1}$ is considered and its transmit power is adjusted to the minimum value by MA. When MAP terminates, we obtain the following results: the network utility is 2.8, the average AP utility is 65.5, and the aggregated throughput is 28 . The three users achieve the bandwidths of 5,9 , and 14 , respectively.

\section{B. Minimize The AP Performance Variance}

After executing MAP, which is the second step of PCAP, all APs may use different power levels to transmit. In this subsection, we propose our algorithm Minimize Performance Variance (MPV) to minimize the variance of the AP utility. The key idea of MPV is similar to that of MAP. At each iteration, the function Minimize_Variance (MV) is called to calculate the current network state that minimizes the variance of the AP utility. This function needs to satisfy the following two conditions:

- Condition 1: The final state of each iteration must be the one that yields the maximum network utility.

- Condition 3: Each iteration should not increase the variance of the AP utility.

Condition 1 is a common requirement for both MA and MV. To satisfy Condition 3, we introduce new variables $\Delta U_{\max }^{\prime a}$ and $\sigma_{u}^{\prime}$ to record the maximum utility deviation and the variance of the AP utility from the previous iteration, respectively.

The operation of MV is executed as follows: First, the recording variables $S^{\prime}, U^{\prime}, \Delta U_{\text {max }}^{\prime a}$, and $\sigma_{u}^{\prime}$ are initialized. Then the AP $k$ with the maximum utility deviation is identified. If $\Delta U_{j}^{a}<0$ and $p_{k}<p_{\max }, p_{k}$ is increased by one level; on the other hand, if $\Delta U_{j}^{a}>0$ and $p_{k}>p_{\min }, p_{k}$ is decreased by one level. With this new transmit power of AP $k$, $S, U, \Delta U_{j}^{a}$ and $\sigma_{u}$ are updated. For both cases, if the network utility is increased and the variance of the AP utility is nonincreased, AP $k$ takes the new transmit power, and the new network information is recorded. Notice the subtle difference for these two cases: AP $k$ takes a lower transmit power as long as the network utility is not decreased for the first case. MV terminates when changing the transmit power of the AP with the maximum utility deviation does not decrease the AP utility variance. This algorithm is described in Alg. 3. A similar analysis as that of MAP indicates that the time complexity of MPV is $O\left(m n^{2} L\right)$, where $m, n$, and $L$ are the number of users, APs, and power levels, respectively.

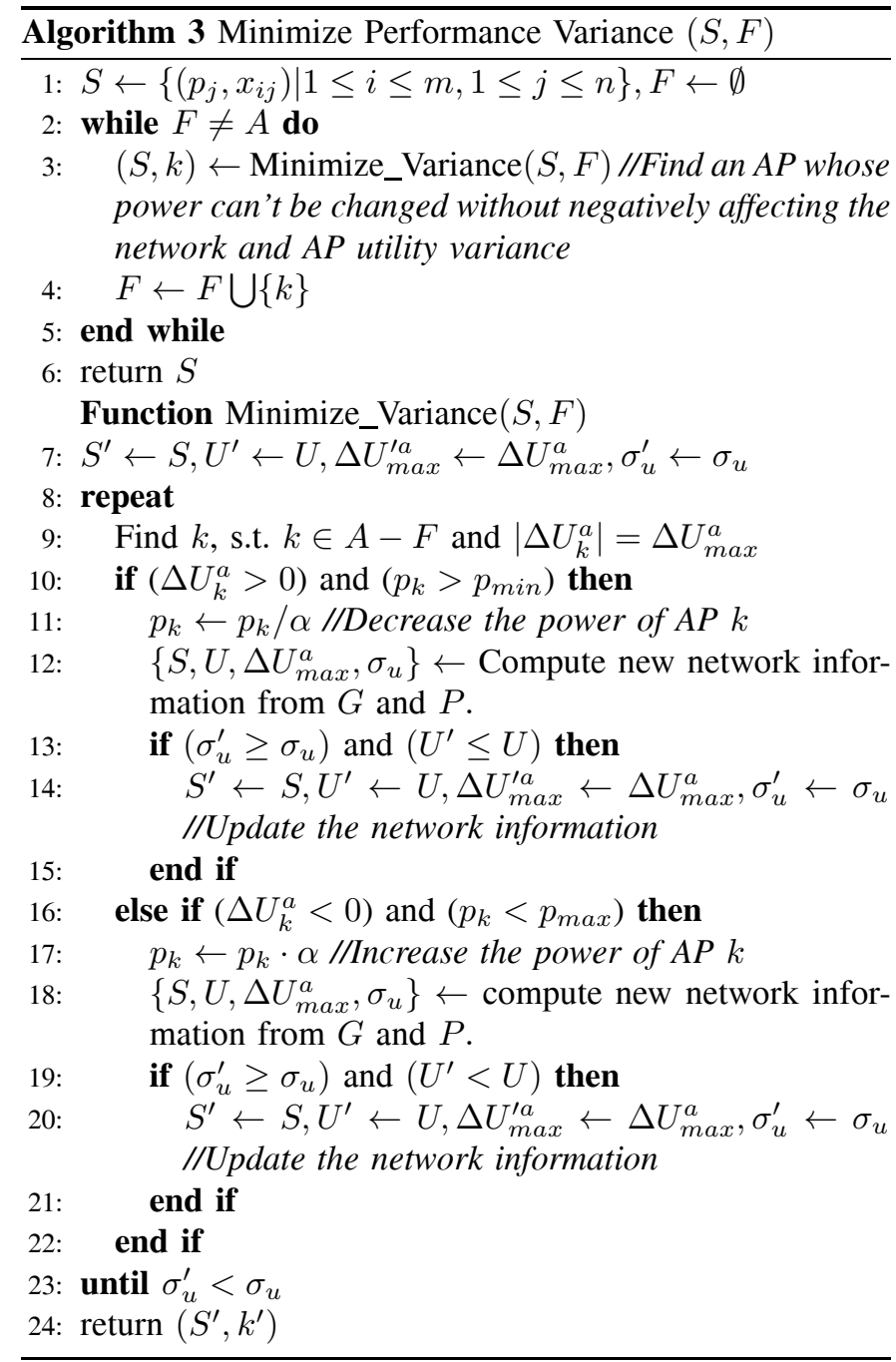

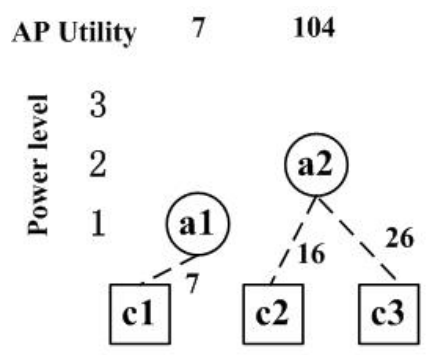

(d) First iteration, $F=\{a 2\}$

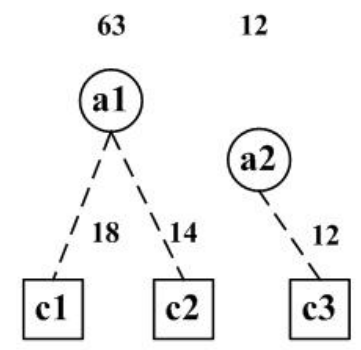

(e) Second iteration, $\mathrm{F}=\{\mathrm{a} 2, \mathrm{a} 1\}$
Fig. 2. An example to illustrate the process of MPV.

We employ the same example (Fig. 1) to illustrate the operation of MPV. After applying MAP, we obtain the results shown in Fig. 1 (c). At this time, the utility deviation of $a_{1}$ is equal to that of $a_{2}$. Since $a_{2}$ has a higher utility, its power is decreased by one level. After the first iteration, the network state is shown in Fig. 2 (d) and $a_{2}$ is added to $F$. Then, we increase the power level of $a_{1}$ and obtain the following final results: the network utility is 2.9 ; the aggregated throughput is 28; and the bandwidths of the three users increase to 9, 7 and 12, respectively. Note that even though the network throughput is not improved after executing MVP, the network utility is increased and the bandwidths allocated to the three 
users are more fair. This result is consistent with the argument stated in [4], in which the network utility is the parameter that better represents the tradeoff between throughput and fairness.

\section{Analysis}

Note that the coverage area of each AP changes as its power is changed. In such a case, a potential concern is that a user may lose its connection to any AP when PCAP is applied. In other words, a user may not reside in the coverage area of any AP due to power control. In the following theorem, we prove that such a possibility does not exist, because PCAP (MAP and MPV) maintains the following invariant characteristic: the network utility is non-decreasing when transmit powers are adjusted.

Theorem 3: PCAP does not change the group of users served by all the APs, although the user-AP association may change.

Proof: We prove this claim by contradiction. Assume there exists a user that losses its association with all APs due to transmit power adjustment when MAP or MPV are executed. According to (4), the network utility becomes negative infinity as the bit rate of the user is decreased to zero. This is impossible because with MAP and MPV, we preserve the invariant that the network utility is non-decreasing, regardless of how the transmit powers are adjusted.

\section{Simulation}

\section{A. Methodology}

We place a total of 20 APs on a 5 by 4 grid, with each $\mathrm{AP}$ on a grid point. The coverage area of each AP is set to 150 meters and the distance between two adjacent APs is set to 100 meters. The maximum and minimum transmit powers of each AP are set to $20 \mathrm{dBm}$ and $10 \mathrm{dBm}$, respectively. These settings are consistent with the values provided by the IEEE 802.11 standard [25]. We arrange 200-350 users to simulate different load scenarios. Assume that all users have the same weight. To consider a more practical scenario, our simulation focuses on a hotspot case where users are randomly positioned in a circle-shaped area with a radius of 100 meters near the center of the 20-AP network.

We use a simple wireless channel model where the bit rate only depends on the SINR. For simplicity, we adopt the values commonly advertised by 802.11 which is shown in Table I. The channel gain is modeled by $g_{i j}=s_{i j} d_{i j}^{-4}$, where $s_{i j}$ is a log-normally distributed shadowing factor, and $d_{i j}$ is the distance between user $i$ and AP $j$. Shadowing factors are generated according to the Viterbi model [26], with $\mu\left(s_{i j}\right)=0 \mathrm{~dB}$ and $\sigma\left(s_{i j}\right)=10 \mathrm{~dB}$. The receiver noise power $N_{0}=-80 \mathrm{dBm}$.

Fairness is quantified by Jain's fairness index [27], which is defined by

$$
J=\frac{\left(\sum_{i=1}^{m} b_{i}\right)^{2}}{m \sum_{i=1}^{m} b_{i}^{2}},
$$

where a larger value of $J$, with $J \in[0,1]$, indicates a better fairness.

We compare PCAP with the following algorithms, which have been summarized in our related work in Section II.

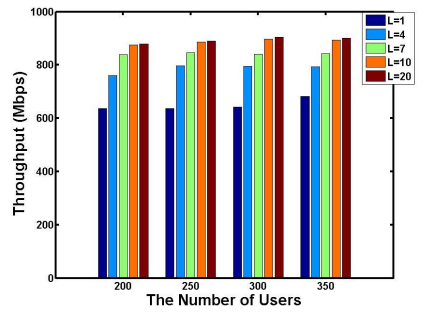

(a) Aggregated network throughput

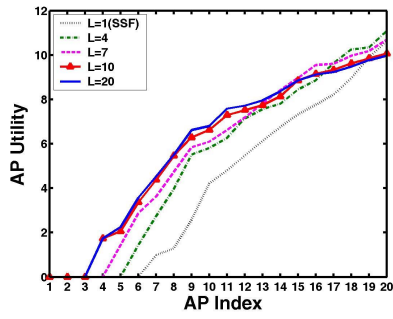

(b) AP utility with 300 users
Fig. 3. The impact of $L$.

TABLE II

THE STATISTICS OF THE RESULTS WHEN $L$ VARIES (300 users)

\begin{tabular}{|c|c|c|c|c|c|}
\hline $\begin{array}{c}\text { Power } \\
\text { Levels }(L)\end{array}$ & $\begin{array}{c}\text { Average AP } \\
\text { Utility }\left(\overline{U^{a}}\right)\end{array}$ & $\begin{array}{c}\text { AP Utility } \\
\text { Variance } \\
\left(\sigma_{u}\right)\end{array}$ & $\begin{array}{c}\text { Total } \\
\text { Network } \\
\text { Utility }\end{array}$ & $\begin{array}{c}\text { Jain's } \\
\text { Fairness } \\
\text { Index }\end{array}$ & $\begin{array}{c}\text { Average } \\
\text { Power } \\
(\mathrm{dBm})\end{array}$ \\
\hline$L=1$ & $1.11 \times 10^{9}$ & $27.31 \times 10^{9}$ & 79.59 & 0.37 & 20.00 \\
$L=4$ & $1.36 \times 10^{9}$ & $11.24 \times 10^{9}$ & 106.80 & 0.82 & 18.19 \\
$L=7$ & $2.57 \times 10^{9}$ & $9.48 \times 10^{9}$ & 113.60 & 0.85 & 17.68 \\
$L=10$ & $3.82 \times 10^{9}$ & $2.98 \times 10^{9}$ & 117.42 & 0.90 & 17.15 \\
$L=20$ & $4.10 \times 10^{9}$ & $2.36 \times 10^{9}$ & 119.00 & 0.90 & 17.03 \\
\hline
\end{tabular}

1) MARL: a power control algorithm based on the monotonic optimization [15].

2) Spectral Radius (SR): a fair transmission rate allocation scheme via power control based on the spectral radius estimation of normalized link gain matrix [12].

3) Strongest Signal First (SSF): the default user-AP association of 802.11.

The metrics to quantify the performances of the algorithms mentioned above include the total utility, the aggregated throughput, Jain's fairness index, and power consumption.

\section{B. Numerical Results}

We report our simulation results in this section. All the results are the averages of 30 runs.

First, we investigate the impact of $L$, the number of power levels, on the performance of our algorithm PCAP. The aggregated network throughput $v s$. the number of users are reported in Fig. 3(a). We observe that with the increase in the number of power levels, the aggregated network throughput consistently increases when $L=1,4,7$ and 10 . Nevertheless, when $L=20$, the aggregated throughput is close to that of $L=10$, which indicates that the benefit introduced by transmit power control is limited when there are too many levels of transmit power. Note that the trends of aggregated network throughput enhancements are similar when the number of users varies from 200 to 350. In fact, for each transmit power level, the aggregated throughput does not decrease when the number of users increases from 200 to 350. This clearly demonstrates the ability of PCAP to balance the user-AP association and to mitigate the intensity of resource competition by controlling the AP transmit powers.

The per-AP utility and statistics information with 300 users are reported in Fig. 3(b) and Table II, respectively. Note that we also conduct simulation studies when there are 200, 250, and 350 number of users and obtained very similar results.

Fig. 3(b) illustrates the per-AP utility presented in logarithmic coordinates when the AP indices are sorted in the 


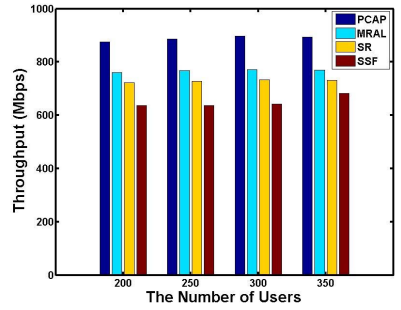

(a) Aggregated network throughput

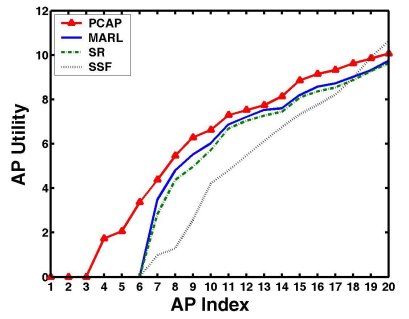

(b) AP utility with 300 users
Fig. 4. The comparison study of different algorithms.

TABLE III

The STATISTICS OF THE RESUlTs (300 users)

\begin{tabular}{|c|c|c|c|c|c|}
\hline Algorithm & $\begin{array}{c}\text { Average AP } \\
\text { Utility }\left(\overline{U^{a}}\right)\end{array}$ & $\begin{array}{c}\text { AP Utility } \\
\text { Variance } \\
\left(\sigma_{u}\right)\end{array}$ & $\begin{array}{c}\text { Total } \\
\text { Network } \\
\text { Utility }\end{array}$ & $\begin{array}{c}\text { Jain's } \\
\text { Fairness } \\
\text { Index }\end{array}$ & $\begin{array}{c}\text { Average } \\
\text { Power } \\
(\mathrm{dBm})\end{array}$ \\
\hline PCAP & $3.82 \times 10^{9}$ & $2.98 \times 10^{9}$ & 117.42 & 0.90 & 17.15 \\
MARL & $2.67 \times 10^{9}$ & $5.00 \times 10^{9}$ & 102.30 & 0.85 & 17.28 \\
SR & $1.46 \times 10^{9}$ & $12.80 \times 10^{9}$ & 99.66 & 0.75 & 18.06 \\
SSF & $1.11 \times 10^{9}$ & $27.31 \times 10^{9}$ & 79.59 & 0.37 & 20.00 \\
\hline
\end{tabular}

non-decreasing order of their utilities. When $L=1$, which corresponds to SSF where all APs transmit with the maximum power, only 14 of the 20 APs serve one or more users while the remaining 6 APs have zero load. For the cases of $L=10$ and $L=20$, the two AP utility curves almost overlap, as the benefit of transmit power control does not increase linearly with $L$ when $L$ is too large, which is consistent with the observations in Fig. 3(a). The number of idle APs decreases as $L$ increases, which indicates that PCAP does make an effort to efficiently manage the network resources.

From Table II, we observe that our algorithm PCAP achieves proportional fairness with a high Jain's fairness index when there are 4 or more transmit power levels. Notice that when $L$ increases, the average AP transmit power decreases, the average AP utility increases, the variance of the AP utility decreases, and the network utility increases. These observations demonstrate the benefit of transmit power control via our algorithm PCAP.

Next, we report the comparison study of the four schemes. The number of transmit power levels in PCAP is set to 10 . The results are summarized in Fig. 4 and Table III.

The aggregated throughput in Mbps of the four algorithms $v s$. the number of users are illustrated in Fig. 4(a). Obviously, PCAP achieves the highest aggregated network throughput while SSF achieves the lowest for all the four load scenarios. In between, MARL performs better than SR.

The per-AP utility and statistics information with 300 users are reported in Fig. 4(b) and Table III, respectively. As shown in Fig. 4(b), SSF forces a user to associate with the AP with the strongest received signal, which increases the intensity of resource competition and reduces the resource utilization (6 APs are idle). On the other hand, SR and MARL allocate the AP transmit powers based on a fixed user-AP association. Since the optimal user-AP association may change when the AP transmit power changes, the improvements of per-AP utilities of MARL and SR are limited. Compared with MARL and SR, PCAP adjusts the AP transmit powers and userAP association simultaneously, and therefore achieves a better result.
Table III reports the statistics of the results of the four algorithms with 300 users in the network. We can see that PCAP performs the best while MARL the second, SR the third, and SSF the fourth, in terms of average AP utility, AP utility variance, total network utility, Jain's fairness index, and the average AP transmit power. These results are consistent with that of Fig. 4(b). Note that our observations clearly demonstrate the relationship among transmit power control, AP utilities, network utility and throughput, and fairness, which further justify the design of our algorithm PCAP. Notice that it is difficult for MARL and SR to improve their user bandwidths as they could not alleviate the resource competition without adjusting the user-AP association. On the contrary, PCAP changes the AP transmit powers and the userAP association simultaneously.

\section{CONCLUSION}

The objective of this paper is to achieve proportional fairness via power control in multi-rate WLANs. Proportional fairness provides a good tradeoff between throughput and fairness, and is used to regulate the bandwidth allocation of users with different transmission rates. We first analyze the relationship between network utility and AP utility according to proportional fairness, i.e, the network utility is maximized when all the APs have the same AP utility for a fixed total AP utility. This relationship is employed to design PCAP, an algorithm to adjust transmit powers for proportional fairness. Note that PCAP adopts the default 802.11 association mechanism to adjust the user-AP association when transmit powers change. Our simulation confirms that PCAP can achieve proportional fair bandwidth allocation and significantly improve the network throughput.

\section{REFERENCES}

[1] A. Akella, G. Judd, S. Seshan, and P. Steenkiste, "Self management in chaotic wireless deployment," Wireless Networks, vol. 13, no. 6, pp. 737-755, Dec. 2007.

[2] G. Tan and J. Guttag, "Time-based fairness improves performance in multi-rate WLANs," in Proc. USENIX Annual Technical Conf., 2004, pp. 23-23.

[3] M. Heusse, F. Rousseau, G. Berger-Sabbatel, and A. Duda, "Performance anomaly of 802.11 b," in Proc. IEEE INFOCOM, 2003, pp. 836843.

[4] F. P. Kelly, "Charging and rate control for elastic traffic," European Trans. Telecommun., vol. 8, no. 1, pp. 33-37, 1997.

[5] A. Banchs, P. Serrano, and H. Oliver, "Proportional fair throughput allocation in multirate IEEE 802.11e wireless LANs," Wireless Networks, vol. 13, no. 5, pp. 649-662, 2007.

[6] A. V. Babu and L. Jacob, "Performance analysis of IEEE 802.11 multirate WLANs: time based fairness vs throughput based fairness," in Proc. IEEE Int. Conf. on Wireless Networks, Communications and Mobile Computing, 2005, pp. 203-208.

[7] D. Bertsekas and R. Gallager, Data Networks. Prentice-Hall, 1987.

[8] W. Li, Y. Cui, S. Wang, and X. Cheng, "Approximate optimization for proportional fair AP association in multi-rate WLANs," in Proc. 5th Int. Conf. WASA, 2010, pp. 36-46.

[9] Y. Bejerano, S.-J. Han, and L. E. Li, "Fairness and load balancing in wireless LANs using association control," IEEE/ACM Trans. Networking, vol. 15, no. 3, pp. 560-573, 2007.

[10] L. E. Li, M. Pal, and Y. R. Yang, "Proportional fairness in multi-rate wireless LANs," in Proc. IEEE INFOCOM, 2008, pp. 1004-1012.

[11] V. P. Mhatre, K. Papagiannaki, and F. Baccelli, "Interference mitigation through power control in high density 802.11 WLANs," in Proc. IEEE INFOCOM, 2007, pp. 535-543.

[12] V. Hasu and V. Koivo, "Fair transmission rate allocation: a power control feasibility approach," in Proc. IEEE ICCS, 2006, pp. 1-5. 
[13] Y. Bejerano and S.-J. Han, "Cell breathing techniques for load balancing in wireless LANs," IEEE Trans. Mobile Computing, vol. 8, no. 6, pp. 735-749, 2009.

[14] S. Wang, M. Liu, X. Cheng, Z. Li, J. Huang, and B. Chen, "Coverage adjustment for load-balancing with an AP service availability guarantee in WLANs," manuscript, 2011.

[15] L. P. Qian and Y. Jun, "Monotonic optimization for non-concave power control in multiuser multicarrier network systems," in Proc. IEEE INFOCOM, 2009, pp. 172-180.

[16] L. Qian, N. Song, D. R. Vaman, X. Li, and Z. Gajic, "Power control and proportional fair scheduling with minimum rate constraints in clustered multihop TD/CDMA wireless ad hoc networks," in Proc. IEEE WCNC, 2006, pp. 763-769.

[17] M. Chiang, C. W. Tan, D. P. Palomar, D. O’Neill, and D. Julian, "Power control by geometric programming," IEEE Trans. Wireless Commun., vol. 6, no. 7, pp. 2640-2651, 2007.

[18] L. Chen and J. Leneutre, "A game theoretic framework of distributed power and rate control in IEEE 802.11 WLANs," IEEE J. Sel. Areas Commun., vol. 26, no. 7, pp. 1128-1137, 2008.

[19] Y. Song, C. Zhang, and Y. Fang, "Joint channel and power allocation in wireless mesh networks: a game theoretical perspective," IEEE J. Sel. Areas Commun., vol. 26, no. 7, pp. 1149-1159, 2008.

[20] M. Rasti, A. R. Sharafat, and B. Seyfe, "Pareto-efficient and goal-driven power control in wireless networks: a game-theoretic approach with a novel pricing scheme," IEEE/ACM Trans. Networking, vol. 17, no. 2, pp. 556-569, 2009.

[21] "High-speed Physical Layer in the $5 \mathrm{GHz}$ Band," 1999.

[22] T. Bu, L. E. Li, and R. Ramjee, "Generalized proportional fair scheduling in third generation wireless data network," in Proc. IEEE INFOCOM, 2006, pp. 1-12.

[23] Available: http://www.cisco.com/en/US/prod/collateral/wireless/ps6302/ ps8322/ps6366/prod $\backslash$ brochure0900aecd805aa860.html

[24] Available: http://www.arubanetworks.com/products/arubaos/ adaptive-radio-management/

[25] "Radio Resource Measurement of Wireless LANs," 2008.

[26] A. J. Viterbi, CDMA: Principles of Spread Spectrum Communication. Addison-Wesley, 1995.

[27] R. Jain, D.-M. Chiu, and W. R. Hawe, "A quantitative measure of fairness and discrimination for resource allocation in shared computer system,” Digital Equipment, Tech. Rep. DEC-TR-301, 1984.

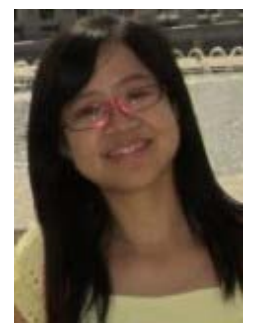

Wei $\mathbf{L i}$ is a first year Ph.D. student of Computer Science at The George Washington University. She received her M.S. degree of Computer Science and B.S. degree of Mathematics from Beijing University of Posts and Telecommunications in 2011 and China Agricultural University in 2008, respectively. She received the unique Best Paper Award in 5th international conference WASA in Aug. 2010. Her research interests include network resource management, algorithm design and analysis.

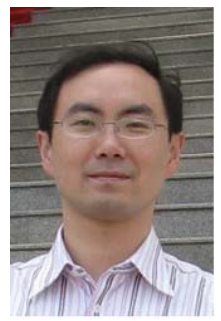

work architecture.

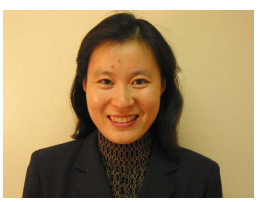

Xiuzhen Cheng received her MS and Ph.D. degrees in computer science from the University of Minnesota - Twin Cities, in 2000 and 2002, respectively. She is an associate professor at the Department of Computer Science, The George Washington University, Washington DC. Her current research interests include cyber-physical systems, wireless and mobile computing, sensor networking, wireless and mobile security, and algorithm design and analysis. She has served on the editorial boards of several technical journals and the technical program committees of various professional conferences/workshops. She also has chaired several international conferences. She worked as a program director for the US National Science Foundation (NSF) from April to October in 2006, and from April 2008 to May 2010. She received the NSF CAREER Award in 2004. She is a member of the IEEE.

Mznah Al-Rodhaan received her BS in Computer Applications (Hon) and MS in Computer Science both from King Saud University, in 1999 and 2003 respectively. In 2009, she received her Ph.D. in Computer Science from the University of Glasgow, Scotland, UK. She is currently working as an assistant professor and vice chair of the Computer Science department, College of Computer and Information Sciences, King Saud University, Riyadh, Saudi Arabia. Moreover, she served in the editorial board of the Ad Hoc Journal (Elsevier) and has participated in several international conferences. Her current research interest includes: Mobile Ad Hoc Networks, Wireless Sensor Networks, Cognitive Networks, Network Security, and High Performance Computing.

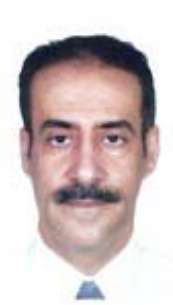

Abdullah Al-Dhelaan received his BS in Statistics (Hon) from King Saud University, in 1982, and his MS and Ph.D. in Computer Science from Oregon State University in 1986 and 1989 respectively. He is currently an Associate Professor of Computer Science, Chairman of the join Ph.D. program, and Director General for the Center for International Collaboration and Visiting Professors, College of Computer and Information Sciences, King Saud University, Riyadh, Saudi Arabia. He has guest edited several special issues for the Telecommunication Journal (Springer), and the International Journal for Computers and Their Applications (ISCA). Moreover, he is currently on the editorial boards of several journals such as Computer Network (Elsevier) and the International Journal of Computers and Their Applications. His current research interest includes: Mobile Ad Hoc Networks, Sensor Networks, Cognitive Networks, Network Security, and High Performance Computing. 